buccal midazolam. Further information about the type and the frequency of training courses (including the refresher courses) being offered to the carers for administration of buccal midazolam would also be documented. There is also data collection pertaining to the timely review of the care plan by the prescribing clinicians and the about the licenced or unlicensed used of midazolam. The quality of epilepsy awareness training is being explored in depth and also the competency of the county council nurses offering the training to the carers shall be analysed under different headings.

Result. Data are currently being collected in Hertfordshire and Essex mental health services.

Conclusion. After the data analysis, the short comings shall be addressed and effective measures shall be put in place to improve the quality of training being provided to the carers and to standardise the practice for prn midazolam prescription.

\section{PLAN standards and writing to patients: quality improvement by audit \\ Edie Shaw ${ }^{1 \star}$, Catherine Adams ${ }^{2}$, Thomas Maclaren ${ }^{2}$ and Fergus Brown ${ }^{3}$ \\ ${ }^{1}$ Foundation Doctor, Imperial College Healthcare NHS Trust; \\ ${ }^{2}$ Consultant Psychiatrist, Chelsea and Westminster Hospital and \\ ${ }^{3}$ Foundation Doctor, Chelsea and Westminster Hospital NHS \\ Foundation Trust \\ ${ }^{*}$ Corresponding author.}

doi: 10.1192/bjo.2021.589

Aims. This quality improvement project aimed to assess the adherence of a hospital psychiatric liaison team's documentation of assessments to the Psychiatric Liaison Accreditation Network (PLAN) standards framework; to identify areas of improvement; to identify barriers to and improve adherence.

Method. Data were extracted from 27 randomly selected patient assessments from $01 / 07 / 2020$ to $31 / 08 / 2020$ and then 27 assessments from $01 / 10 / 2020$ to $30 / 11 / 2020$ for re-audit.

Quantitative data was collected by calculating the percentage of assessments which documented each specific aspect of PLAN standards.

Qualitative data including attitudes specifically towards writing to patients was gathered from 1:1 discussions with members of staff. Interventions between rounds of audit:

Presentation of results of 1 st data collection to team in November 2020 followed by discussion

Emailed instructions to create a template based on PLAN standards for assessments to staff

Lobbied for Cerner access at liaison team office to facilitate use of above

Result. Quantitative - overall improvements were seen in adherence to all aspects of documentation of assessments including collateral history (from $23 \%$ to $67 \%$ ) past medical history (30\% to $70 \%)$ and acknowledging the patient/carer perspective (46\% to $74 \%)$. Some improvement was seen in offering written correspondence to patients ( $0 \%$ to $20 \%$ ).

Qualitative - the majority of comments regarding writing to patients were positive, with no staff members opposing the standard ("it is best practice", "should become a habit"). However, some barriers were identified including increased workload ("requires more editing", "could take a lot more time").

Conclusion. Team adherence to PLAN standards for documentation of assessments was improved through low intensity interventions. Overall adherence was high, however certain areas leave space for improvement. The audit facilitated conversations around writing to patients on discharge, both in the form of formal gathering of qualitative data and informal discussions between staff. Attitudes towards writing these letters were positive and some improvement was seen between audits. Ongoing audit activity aims to further improve adherence and monitor improvements.

\section{Prevalence of pulmonary disease in alcohol and poly} substance abuse

Ghazanfar Sheikh

CGL

doi: 10.1192/bjo.2021.590

Aims. The aim of this study is to identify the prevalence of pulmonary disease or progression of symptoms which can lead to respiratory disease in substance misuse. Identify risk: prescribe a safer substitute treatment (Methadone vs Buprenorphine). Identify combination of different drugs, causing toxicity and damage to lungs.

Background. Smoking and illicit substance misuse is recognized as a cause of pulmonary disease

Method. The study was conducted in 2015 at outpatient addictions service (CAPS/CDC 69 Warwick Road London SW5 9HB). All 50-participants gave consent and data were collected including demographic details, history of substance abuse, route of administration, medical history, Peak Expiratory Flow rate (PEFR). A questionnaire was designed to assess the severity of shortness of breath (SOB), with or without exertion to ascertain the extent of the disease.

Conclusion. 14 males and 3 females achieved 31 to $48.9 \%$ of predicted PEFR. 13 males and 7 females achieved 51.1 to $69.6 \%$ of predicted PEFR. 7 males and 2 females achieved 70.8 to $91.1 \%$ of predicted PEFR. 2 males and 2 females achieved 100 to $114.3 \%$ of predicted PEFR. From the questionnaire on SOB with or without exertion, 8 males and 2 females reported moderate to severe shortness of breath on minimum exertion. 33 males and 11 females reported variable degree of SOB. 4 males and 2 females did not report SOB on reasonable exertion. 23 males and 10 females reported variable degree of SOB at rest, 14 males and 3 females reported no SOB at rest. 34 participants were receiving OST with methadone, 16 were on Buprenorphine. 6 patient treatments were switched to Buprenorphine due to Exacerbated COPD. 47 patients reported using Tobacco from harmful to dependent use. 44 patients reported harmful to dependent use of crack cocaine. 34 patients fulfilled the criteria of harmful use of cannabinoids. 33 patients were diagnosed with harmful to dependent use of alcohol. 10 patients reported heavy use of solvents. 2 patients reported harmful use of Party drugs. Many patients reported a history of recurrent chest infections 3-6 times a year, Asthma, COPD, Emphysema, Pneumonia and 2 were treated for Tuberculosis. 1/ 3 patients have a diagnosis of hypertension and IHD, liver disease and portal hypertension

Quality improvement project: to improve adherence to DVLA (driving and vehicle liscensing agency) guidance in the tyrone \& fermanagh hospital acute inpatients ward

Vivian Sing ${ }^{1 \star}$, Chad Ballantine ${ }^{2}$ and Peter Hackett ${ }^{2}$

${ }^{1}$ Ulster Hospital and ${ }^{2}$ Tyrone and Fermanagh Hospital ${ }^{\star}$ Corresponding author.

doi: 10.1192/bjo.2021.591 